\title{
Variational approach to the scattering of charged particles by a many-electron system
}

\author{
V. U. Nazarov, ${ }^{1}$ S. Nishigaki, ${ }^{2}$ J. M. Pitarke, ${ }^{3,4}$ and C. S. Kim ${ }^{1}$ \\ ${ }^{1}$ Department of Physics and Institute for Condensed Matter Theory, Chonnam National University, Gwangju 500-757, Korea \\ ${ }^{2}$ Kyushu Institute of Technology, Sensui-cho 1-1, Tobata, Kitakyushu 804-8550, Japan \\ ${ }^{3}$ Materia Kondentsatuaren Fisika Saila, Zientzi Fakultatea, Euskal Herriko Unibertsitatea, 644 Posta Kutxatila, E-48080 Bilbo, \\ Basque Country, Spain \\ ${ }^{4}$ Donostia International Physics Center and Centro Mixto CSIC-UPV/EHU, Donostia, Basque Country, Spain
}

(Received 29 October 2004; revised manuscript received 10 December 2004; published 29 March 2005)

\begin{abstract}
We report a variational approach to the nonlinearly screened interaction of charged particles with a manyelectron system. This approach has been developed by introducing a modification of the Schwinger variational principle of scattering theory, which allows one to obtain nonperturbative scattering cross sections of moving projectiles from the knowledge of the linear and quadratic density-response functions of the target. Our theory is illustrated with a calculation of the energy loss per unit path length of slow antiprotons moving in a uniform electron gas, which shows good agreement with a fully nonlinear self-consistent Hartree calculation. Since available self-consistent calculations are restricted to low heavy-projectile velocities, we expect our theory to have applications to a variety of processes where nonlinear screening plays an important role.
\end{abstract}

DOI: 10.1103/PhysRevB.71.113105

PACS number(s): 71.45.Gm, 11.80.Fv

The interaction of external charges with solid targets is a problem of both fundamental and practical interest in contemporary physics. ${ }^{1,2}$ Since the targets of interest, either bulk solids, surfaces, or structures of lower dimension, typically consist of many interacting electrons, a remarkable progress has been achieved along the lines of the so-called dielectric perturbative formulation of scattering. This formulation utilizes density-response functions of many-body systems to characterize their dynamical reaction to external perturbations. ${ }^{2,3}$

Linear-response theory provides a qualitative description of the energy loss per unit path length of external particles of charge $Z_{1}$ interacting with solid targets, i.e., the so-called stopping power (SP) of the solid. However, it yields SP that is proportional to $Z_{1}^{2}$ and cannot, therefore, account for the existing differences between the ranges of positive and negative pions ${ }^{4}$ and the slowing of protons and antiprotons. ${ }^{5,6}$ The quadratic-response treatment considerably improves the description of the interaction of external charges with solid targets, ${ }^{7,8}$ and yields a $Z_{1}^{3}$ correction to the energy loss that accurately accounts for the measured energy loss of protons and antiprotons in the high-velocity regime. ${ }^{9,10}$ Considerable progress has been achieved recently in the framework of quadratic-response theory ${ }^{11,12}$ however, at low velocities this theory is only able to quantitatively account for the strong influence of unit-charge projectiles $\left(Z_{1}= \pm 1\right)$ in the case of high-density targets.

Another approach to investigate the interaction of external charges with a many-electron system is based on the ordinary formulation of potential-scattering theory. In this approach, ${ }^{13-17}$ SP for a heavy particle is determined in the low-velocity limit from the knowledge of the scattering phase shifts corresponding to a static nonlinearly screened potential. These potential-scattering calculations include all orders in the projectile charge; however, they have the limitation of being restricted to low velocities $\left(v \ll v_{F}, v_{F}\right.$ being the Fermi velocity) of heavy projectiles moving in bulk materials.
In the case of few-body scattering, variational methods of the Schwinger type are known to provide nonperturbative representations of the collision matrix, ${ }^{18}$ which in the case of the scattering of external charges would not be restricted to low velocities and heavy projectiles. However, the direct application of these methods to solid targets composed with a large number of electrons is not feasible, since it requires the knowledge of the many-particle ground and excited states of the target.

In this paper, we put forward a theory that reconciles the dielectric formulation of scattering with the variational method. To do this, we introduce a modification of the Schwinger variational principle of scattering theory to provide a nonperturbative representation of the scattering cross section of external charges interacting with a many-electron system. This method does not require the knowledge of the many-particle ground and excited states of the target; instead, only the linear and quadratic density-response functions are needed, which have been previously obtained in the framework of time-dependent density-functional theory (TDDFT). ${ }^{19}$

Because of the quite general nature of this approach, it can be readily applied to the investigation of a variety of processes involving the inelastic scattering of charged particles by many-electron targets, such as the SP for moving ions, electron and positron energy-loss spectroscopy, inelastic low-energy electron diffraction, and hot-electron dynamics. As our formula only requires the knowledge of the two lowest-order density-response functions of the target, the implementation of this approach has a computational cost equal to that of available quadratic-response theories.

Let us consider the scattering of a charge by an arbitrary many-electron target, in which the target is scattered between its ground $|0\rangle$ and excited $|n\rangle$ states. We assume that the initial and final states $\Psi_{i}=\left|\mathbf{p}_{i}, 0\right\rangle$ and $\Psi_{f}=\left|\mathbf{p}_{f}, n\right\rangle$ of the projectile-target interacting system contain a free particle of charge $Z_{1}$ and momentum $\mathbf{p}_{i}$ and $\mathbf{p}_{f}$, respectively. According 
to the bilinear form of the Schwinger variational principle, ${ }^{18}$ the functional

$$
\left[T_{f i}\right]=\left\langle\Psi_{f}^{-}|V| \Psi_{i}\right\rangle+\left\langle\Psi_{f}|V| \Psi_{i}^{+}\right\rangle-\left\langle\Psi_{f}^{-}\left|V-V G_{0}^{(+)} V\right| \Psi_{i}^{+}\right\rangle
$$

is stationary under the variation of the trial (unknown) eigenstates $\Psi_{f}^{+}$and $\Psi_{i}^{-}$of the full interacting Hamiltonian and at its stationary point gives the exact transition-matrix elements between the (known) initial $\Psi_{i}$ and final $\Psi_{f}$ states. Here $V$ is the Coulomb interaction between the projectile and the target, and $G_{0}^{(+)}$is the Green function associated to the Hamiltonian of the projectile-target system without the mutual interaction $V$.

The fractional form of the Schwinger variational principle is obtained by substituting (see Ref. 18, p. 412)

$$
\Psi_{i}^{+} \rightarrow A \Psi_{i}^{+}, \quad \Psi_{f}^{-} \rightarrow B \Psi_{f}^{-}
$$

in Eq. (1) and treating the coefficients $A$ and $B$ as variational parameters. If one then returns to Eq. (1) and uses the free states $\Psi_{i, f}$ for the trial functions, one finds

$$
T_{f i}=T_{f i}^{(1)} /\left(1-T_{f i}^{(2)} / T_{f i}^{(1)}\right),
$$

where $T_{f i}^{(1)}=\left\langle\Psi_{f}|V| \Psi_{i}\right\rangle$ and $T_{f i}^{(2)}=\left\langle\Psi_{f}\left|V G_{0}^{(+)} V\right| \Psi_{i}\right\rangle$ represent the first two Born transition amplitudes.

Equation (3) has proven very useful in atomic scattering, where the $|0\rangle$ and $|n\rangle$ states of the target can be known, at least approximately. However, in the case of solid targets the many-body ground and excited states are difficult to know. In order to find a representation of the scattering cross section of external charges interacting with a many-electron system in terms of density-response functions, which can be calculated from the knowledge of one-electron states, we first construct the differential cross section for the projectile to be scattered between the free-particle states of momenta $\mathbf{p}$ and $\mathbf{p - k}$ (we use atomic units throughout) ${ }^{18}$

$$
d \sigma / d \mathbf{k}=\left(16 \pi^{4} / v\right) \sum_{n}\left|T_{f n, i 0}\right|^{2} \delta\left(\omega-\omega_{n 0}\right),
$$

where the sum is extended over a complete set of eigenstates of the target, $\omega_{n 0}$ are the excitation energies of the target, $\omega=\mathbf{k} \cdot \mathbf{v}-\mathbf{k}^{2} / 2 M, M$ is the projectile mass, and $\mathbf{v}=\mathbf{p} / M$ is the projectile initial velocity. We then introduce the functional

$$
\begin{aligned}
{[d \sigma / d \mathbf{k}]=} & \left(16 \pi^{4} / v\right) \sum_{n} \mid\left\langle\Psi_{f n}^{-}|V| \Psi_{i 0}\right\rangle+\left\langle\Psi_{f n}|V| \Psi_{i 0}^{+}\right\rangle-\left\langle\Psi_{f n}^{-}\right| V \\
& -\left.V G_{0}^{(+)} V\left|\Psi_{i 0}^{+}\right\rangle\right|^{2} \delta\left(\omega-\omega_{n 0}\right)
\end{aligned}
$$

we compare this functional with that of Eq. (1), and noting that a linear combination of the squares of the absolute value of a stationary quantity is a stationary quantity, we conclude that the functional of Eq. (5) gives the exact cross section of Eq. (4) at its stationary point with respect to the variation of the trial states $\Psi_{i 0}^{+}$and $\Psi_{f n}^{-}$. The variational principle based on the functional of Eq. (5) is the "global" analog (summed over all final states of the target) of the corresponding "local" variational principle based on the functional of Eq. (1) for the transition to a particular state. These two principles are both exact and equivalent to each other.
In order to obtain the fractional form of the global variational principle, we first apply the substitution (2) to the functional (5), and we then approximate the trial functions by the free states. Hence, the problem reduces to finding the stationary value of the functional

$$
\begin{aligned}
{[d \sigma / d \mathbf{k}]=} & 16 \pi^{4} / v \\
& \times \sum_{n}\left|(A+B-A B) T_{f n, i}^{(1)}+A B T_{f n, i}^{(2)}\right|^{2} \delta\left(\omega-\omega_{n 0}\right)
\end{aligned}
$$

with respect to the variational parameters $A$ and $B$. Equation (6) can be rewritten as

$$
[d \sigma / d \mathbf{k}]=(A+B-A B)^{2} R_{2}+A B(A+B-A B) R_{3}+A^{2} B^{2} R_{4} .
$$

Here, $R_{2}, R_{3}$, and $R_{4}$ represent contributions to the differential cross section that are proportional to $Z_{1}^{2}, Z_{1}^{3}$, and $Z_{1}^{4}$, respectively, which can be obtained from the knowledge of the corresponding density-response functions of the target, as shown in Ref. 12.

In what follows, we neglect the last term of Eq. (7) (see the discussion below). Solving the variational problem for the function of Eq. (7) with respect to the parameters $A$ and $B$, we find its stationary value as

$$
d \sigma / d \mathbf{k}=R_{2} f\left(R_{3} / R_{2}\right)
$$

where

$$
\begin{aligned}
f(x)= & {\left[16-32 x-56 x^{2}+72 x^{3}-27 x^{4}+(2-x)(4-4 x\right.} \\
& \left.\left.+9 x^{2}\right)^{3 / 2}\right] / 32(1-x)^{3} .
\end{aligned}
$$

Equation (8) is the global analog of the local Eq. (3). If the quantity $x=R_{3} / R_{2}$ is small, we may expand the expression (9) and obtain

$$
d \sigma / d \mathbf{k}=R_{2}+R_{3}+\cdots,
$$

which agrees with the Born series through second order in the interaction between the projectile and the target.

In the case of antiprotons $\left(Z_{1}=-1\right)$ moving slowly in a metal, the $Z_{1}^{3}$ contribution to the scattering cross section $R_{3}$, which is negative, can overcome for low-density metallic targets the positive $Z_{1}^{2}$ contribution $R_{2}$, thereby leading to a physically meaningless negative scattering cross section. In contrast, our nonperturbative differential cross section (8) is positively defined, as shown in Fig. 1 where the function $f(x)$ of Eq. (9) is plotted.

We illustrate our theory with a calculation of the SP $[-(d E / d x)]$ for slow protons and antiprotons moving in a uniform electron gas (EG) of density $n_{0}$ characterized by the density parameter $r_{s}=(3 / 4 \pi) n_{0}^{-1 / 3}$. At low velocities the projectile-target Coulomb interaction is relatively strong, so this represents an unfavorable situation for a linear or quadratic perturbative approach.

The SP is obtained by multiplying the differential cross section $(d \sigma / d \mathbf{k})$ by the energy transfer $\omega$ and integrating over the momentum transfer $\mathbf{k}$. As the mass $M$ of our projectile is much larger than the electron mass, $\omega \sim \mathbf{k} \cdot \mathbf{v}$ and one writes 


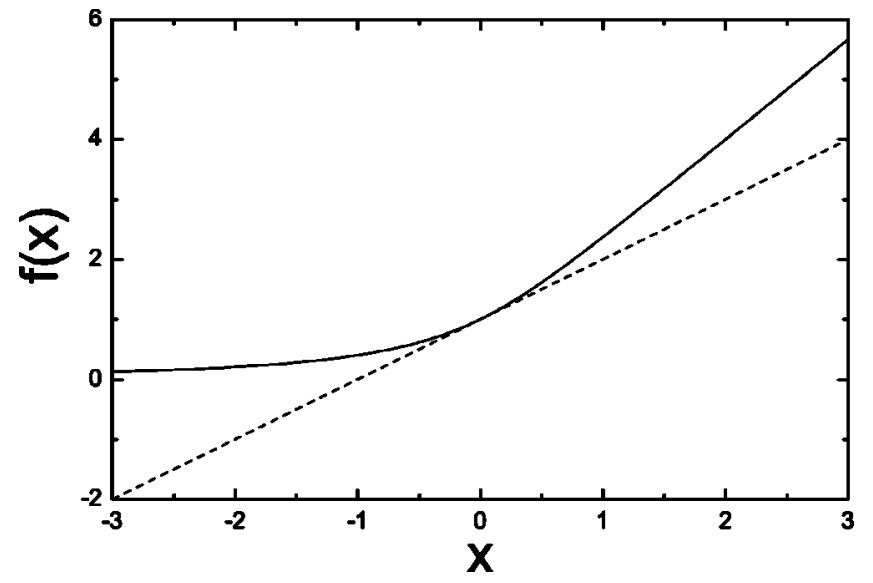

FIG. 1. $f(x)$ of Eq. (9) (solid line). If $x$ is small then $f(x)=1+x+\ldots$ (dashed line), which yields Eq. (10).

$$
-d E / d x=(1 / \Omega) \int d \mathbf{k} \mathbf{v} \cdot \mathbf{k}(d \sigma / d \mathbf{k}),
$$

where $\Omega$ is the normalization volume. We have evaluated the differential cross section of Eq. (8) from the knowledge of the linear and quadratic density-response functions of the uniform EG, which we have calculated in the random-phase approximation (RPA). ${ }^{10}$ For slow projectiles $\left(v<v_{F}\right)$, the SP is found to be proportional to the projectile velocity $v$.

In Fig. 2, the variational SP of a uniform EG for slow protons and antiprotons (thick solid lines) is compared to the corresponding quadratic SP [obtained from Eq. (10)] (dashed lines) and accurate fully nonlinear potential-scattering calculations that we have carried out along the lines of Refs. 14 and 15 but with exchange-correlation (xc) excluded (chained lines). In the high-density limit $\left(r_{s} \rightarrow 0\right)$, the Born series quickly converges and all calculations coincide. However, as

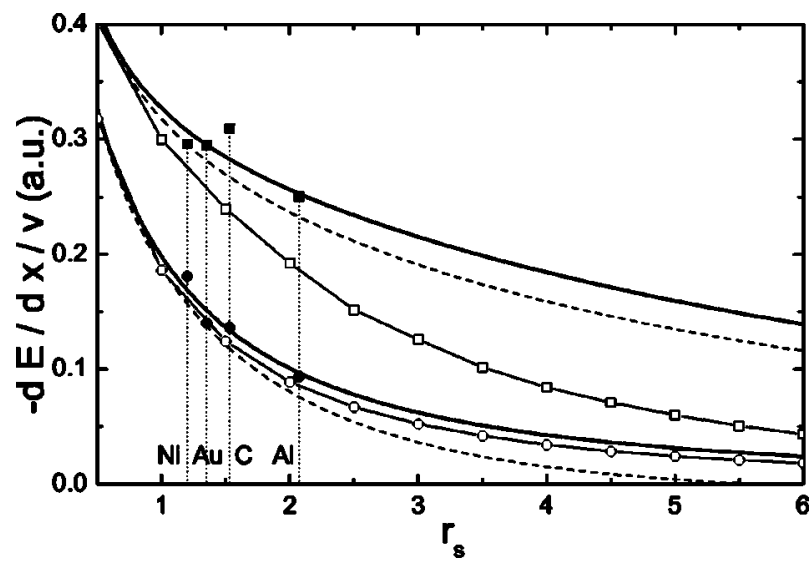

FIG. 2. Variational (solid lines) and quadratic (dashed lines) SP of a uniform EG for slow antiprotons (lower curves) and protons (upper curves), divided by the projectile velocity, as a function of $r_{s}$. The chained lines with circles (squares) represent fully nonlinear Hartree calculations for antiprotons (protons), performed along the lines of Refs. 14 and 15 but with xc excluded. The circles (squares) show the experimental results for antiprotons (protons) reported in Refs. 5 and 6. the electron density decreases, a correct description of nonlinear interactions requires to go beyond quadratic-response theory.

In the case of antiprotons, quadratic-response calculations (lower dashed line of Fig. 2) overestimate the negative nonlinear contribution to the SP (see also Fig. 1), which for $r_{s}>5.5$ becomes larger in magnitude than the linear $\left(Z_{1}^{2}\right)$ term. In contrast, the variational SP (lower thick solid line) is positive for all electron densities and shows good agreement with the fully nonlinear potential-scattering calculation that we have performed by solving self-consistently the Hartree equation of a static antiproton (chained line with circles). Since the Hartree SP is obtained from accurate phase-shift calculations to all orders in $Z_{1}$, the agreement between variational and Hartree calculations gives us confidence in the accurateness of the variational approach.

In the case of protons, our variational SP (upper thick solid line of Fig. 2) overestimates the potential-scattering self-consistent Hartree calculations (chained line with squares) even more than within quadratic-response theory (upper dashed line). This is because in its present (initial) form our theory is only applicable to point charges that do not support bound states or resonances. ${ }^{23}$ Protons in a uniform EG are known to support either a bound state (at $r_{s}>2$ ) or a resonance below the Fermi level, ${ }^{14}$ which can be inferred by the behavior of our calculated scattering phase shifts. Electrons accommodated in either a bound state or a resonance efficiently screen the projectile, thereby decreasing the SP, as shown in Fig. 2.

The first unambiguous evidence for a velocityproportional electronic SP of solid targets has been reported recently, with measurements of the energy loss of slow antiprotons in $\mathrm{Ni}, \mathrm{Au}, \mathrm{C}$, and $\mathrm{Al} .{ }^{5,6}$ At low velocities, the energy loss of ions in metals is mainly due to the slowing by valence electrons, which can be approximately described with the use of a uniform EG. ${ }^{20}$ Hence, we have also plotted in Fig. 2 the friction coefficients $-(d E / d x) / v$ obtained from the slowantiproton energy-loss measurements of Refs. 5 and 6, at $r_{s}=1.20,1.35,1.53$, and 2.07 , corresponding to the average density of valence $3 d^{8} 4 s^{2}, 5 d^{10} 6 s^{1}, 2 s^{2} 2 p^{2}$, and $3 s^{2} 3 p^{1}$ electrons in $\mathrm{Ni}, \mathrm{Au}, \mathrm{C}$, and $\mathrm{Al}$, respectively. The variational and Hartree calculations for antiprotons are considerably closer to experiment (solid circles) than their quadratic counterpart. Our variational calculations for protons are also close to experiment; however, this might be due to a cancelation of the impact of bound and resonance states with the effect of xc not included in our approach. The xc effects were included in Refs. 13-15 in the framework of density-functional theory (DFT), ${ }^{21}$ and they can be included in our variational procedure by going beyond the RPA in the description of the density-response functions of the solid. Work in this direction utilizing the recently obtained quadratic densityresponse function from TDDFT with $x c$ included $^{22}$ is now in progress.

Although an additional approximation of omitting the last term in Eq. (7) has been made, we note that by including it, a more general analytical formula for the differential cross section would be obtained. However, while the terms $R_{2}$ and $R_{3}$ require the knowledge of the linear and quadratic densityresponse functions, respectively, which are available from 
the literature for a number of systems of physical importance, $R_{4}$ is related to the cubic response function, which has not been determined as yet. Although the generalization of the present theory in this direction is conceptually straightforward, for the sake of simplicity (and implementability in calculations at present) we defer it until later publications.

In conclusion, we have reported a nonperturbative variational approach to the nonlinearly screened interaction of charged particles with a many-electron system, which goes beyond the conventional linear and quadratic theories. This approach has been developed by introducing a modification of the Schwinger variational principle of scattering theory, which allows one to obtain nonperturbative scattering cross sections from the knowledge of the linear and quadratic density-response functions of the target. Our approach, which includes contributions to all orders in the projectiletarget Coulomb interaction and agrees with the Born series through second order, represents a considerable improvement over the quadratic theory $\left(Z_{1}^{3}\right)$ approximation; in particular, our variational differential cross section is positively defined, which is known not to be the case for the quadratic theory.

We have illustrated our theory with a calculation of the stopping power of uniform EG for slow protons and antiprotons, which, in the case of antiprotons, shows good agreement with fully nonlinear Hartree calculations. Our calculations indicate that by going beyond quadratic theory the variational procedure considerably improves the agreement with recent measurements of the stopping power of $\mathrm{Ni}, \mathrm{C}$, $\mathrm{Al}$, and $\mathrm{Au}$ for slow antiprotons, though xc effects still need to be taken into account. In the case of protons, the presence of bound states and resonances also needs to be incorporated into our theory. Since self-consistent Hartree calculations have the limitation of being restricted to low heavy-projectile velocities $\left(v \ll v_{F}\right)$, we expect our theory to have applications in the investigation of a variety of processes involving the inelastic scattering of charges by many-electron targets.

We thank P. M. Echenique and E. Zaremba for helpful discussions. V.U.N. and C.S.K. acknowledge support by the Korea Research Foundation through Grant No. KRF-2003015-C00214. J.M.P. acknowledges partial support by the UPV/EHU, the Basque Hezkuntza, Unibertsitate eta Ikerketa Saila, and the Spanish MCyT.
${ }^{1}$ H. Ibach and D. L. Mills, Electron Energy Loss Spectroscopy and Surface Vibrations (Academic Press, New York, 1982).

${ }^{2}$ P. M. Echenique, F. Flores, and R. H. Ritchie, Solid State Phys. 43, 229 (1990).

${ }^{3}$ A. Liebsch, Electronic Excitations at Metal Surfaces (Plenum, New York, 1997).

${ }^{4}$ W. H. Barkas, W. Birnbaum, and F. M. Smith, Phys. Rev. 101, 778 (1956).

${ }^{5}$ S. P. Møller, A. Csete, T. Ichioka, H. Knudsen, U. I. Uggerhøj, and H. H. Andersen, Phys. Rev. Lett. 88, 193201 (2002).

${ }^{6}$ S. P. Møller, A. Csete, T. Ichioka, H. Knudsen, U. I. Uggerhøj, and H. H. Andersen, Phys. Rev. Lett. 93, 042502 (2004).

${ }^{7}$ C. C. Sung and R. H. Ritchie, Phys. Rev. A 28, 674 (1983).

${ }^{8}$ C. D. Hu and E. Zaremba, Phys. Rev. B 37, 9268 (1988).

${ }^{9}$ J. M. Pitarke, R. H. Ritchie, P. M. Echenique, and E. Zaremba, Europhys. Lett. 24, 613 (1993).

${ }^{10}$ J. M. Pitarke, R. H. Ritchie, and P. M. Echenique, Phys. Rev. B 52, 13883 (1995).

${ }^{11}$ T. del Rio Gaztelurrutia and J. M. Pitarke, J. Phys. A 34, 7607 (2001)

${ }^{12}$ V. U. Nazarov and S. Nishigaki, Phys. Rev. B 65, 094303 (2002).

${ }^{13}$ P. M. Echenique, R. M. Nieminen, and R. H. Ritchie, Solid State
Commun. 37, 779 (1981).

${ }^{14}$ P. M. Echenique, R. M. Nieminen, J. C. Ashley, and R. H. Ritchie, Phys. Rev. A 33, 897 (1986).

${ }^{15}$ I. Nagy, A. Arnau, P. M. Echenique, and E. Zaremba, Phys. Rev. B 40, R11 983 (1989).

${ }^{16}$ K. Ladanyi, I. Nagy, and B. Apagyi, Phys. Rev. A 45, 2989 (1992).

${ }^{17}$ A. Krakovsky and J. K. Percus, Phys. Rev. B 52, R2305 (1995).

${ }^{18}$ C. J. Joachain, Quantum Collision Theory (North-Holland, Amsterdam, 1975).

${ }^{19}$ M. Petersilka, U. J. Gossmann, and E. K. U. Gross, Phys. Rev. Lett. 76, 1212 (1996).

${ }^{20}$ I. Campillo, J. M. Pitarke, and A. G. Eguiluz, Phys. Rev. B 58, 10307 (1998).

${ }^{21}$ W. Kohn and L. J. Sham, Phys. Rev. 140, A1133 (1965).

${ }^{22}$ V. U. Nazarov, J. M. Pitarke, C. S. Kim, and Y. Takada, J. Phys.: Condens. Matter 16, 8621 (2004).

${ }^{23}$ This difficulty can be overcome by replacing the plane-wave trial wave functions that we have used in our variational calculations by wave functions appropriate to the description of composite projectiles. 